\title{
BEHAVIOR OF SECOND ORDER NONLINEAR DIFFERENTIAL EQUATIONS
}

\author{
RINA LING \\ Department of Mathematics \\ California State University \\ Los Angeles, California 90032
}

(Received November 29, 1977 and in revised form March 31, 1978)

ABSTRACT. Qualitative behavior of second order nonlinear differential equations with variable coefficients is studied. It includes properties such as positivity, number of zeroes, oscillatory behavior, boundedness and monotonicity of the solutions.

1. INTRODUCTION.

Second order nonlinear differential equations of the form

$$
y(t)+p(t) y(t)+q(t) y^{n}(t)=0
$$

where $\mathrm{n}$ is an integer $\geq 2$, occur in many physical problems, such as the massspring systems and satellite (see Ames [1], Mclachlan [2] and Struble [3]) and nuclear energy distribution (see Canosa and Cole $[4,5]$ ).

In this work, qualitative behavior of real-valued solutions of (1.1) is studied. With certain conditions on the coefficients $p(t)$ and $q(t)$, and $n$, properties such as positivity, number of zeroes, boundedness and monotonicity 
are obtained. It would be assumed that the coefficients and their derivatives are continuous real-valued functions on the interval of interest. The work is divided into four parts; the first part deals with the case of $p(t)<0$ and $q(t)<0$, the second part with the case of $p(t)<0$ and $q(t)>0$, the third part with $p(t)>0$ and $q(t)<0$ and the fourth part with $p(t)>0$ and $q(t)>0$. Papers in the past, Skidmore [6], Abramovich [7], Rankin [8], and Grimmer and Patula [9] have studied behavior of second order linear differential equations. Nonlinear differential equations have been investigated in Chen [10] and Chen, Yeh and Yu [11], the former is on oscillatory behavior of bounded solutions and the latter on asymptotic behavior of solutions. The results here are of a different nature and are independent of theirs.

2. CASE OF $p(t)<0$ AND $q(t)<0$.

THEOREM 2.1. If (1) $p(t)<0, t \geq 0$, (2) $q(t)<0, t \geq 0$ and (3) $n$ is odd, then either $y(t)>0, t>0$ or $y(t)<0, t>0$. The graph is concave upward for $\mathrm{y}>0$, and concave downward for $\mathrm{y}<0$.

PROOF. Equation (1.1) can be written in the form

$$
\ddot{y}+\left(p(t)+q(t) y^{n-1}\right) y=0
$$

Let $z(t)$ be real-valued and satisfy the linear equation

$$
\ddot{z}+p(t) z=0 \text {. }
$$

By a theorem in Hartman $[12$, p. 346-347], $z(t)$ has no zero. Since $n-1$ is even and $q(t)<0$, we have

$$
p(t)+q(t) y^{n-1}<p(t)
$$

and by Sturm First Comparison Theorem, $z(t)$ has at least a zero on $(0, \infty)$, if $y$ has a zero on $(0, \infty)$, a contradiction.

The concavity of the graph follows from (1.1) written in the form 


$$
\ddot{y}=-p(t) y-q(t) y^{n} \text {. }
$$

The case of $\mathrm{n}$ being even is considered in the following theorem.

THEOREM 2.2. If (1) $p(t)<0, t \geq 0$, (2) $q(t)<0, t \geq 0$ and (3) $n$ is even, then $y(t) \leq 0$, for all $t \geq 0$.

PROOF. Since $q(t)<0$ and $n$ is even, we have

$$
\ddot{y}+p(t) y+q(t) y^{n} \leq \ddot{y}+p(t) y,
$$

therefore

$$
0 \leq \ddot{y}+p(t) y
$$

and by Bellman and Kalaba [13, p. 67], $y(t) \leq 0$ for all $t \geq 0$.

3. CASE OF $p(t)<0$ AND $q(t)>0$.

THEOREM 3.1. If (1) $p(t)<0, t \geq 0$, (2) $q(t)>0, t \geq 0$ and (3) $n$ is even, then $y(t) \geq 0$, for all $t \geq 0$.

PROOF. METHOD 1. If in (1.1), we let $y(t)=-z(t)$, then the equation becomes

$$
-\ddot{z}-p(t) z+q(t)(-1)^{n} z^{n}=0,
$$

since $n$ is even,

$$
\ddot{z}+p(t) z-q(t) z^{n}=0
$$

and by Theorem 2.2, $z(t) \leq 0$ for all $t \geq 0$. Therefore $y(t) \geq 0$ for all $t \geq 0$.

METHOD 2. Since $q(t)>0$ and $n$ is even, we have

$$
\ddot{y}+p(t) y \leq \ddot{y}+p(t) y+q(t) y^{n},
$$

therefore

$$
\ddot{y}+p(t) y \leq 0
$$

and by Bellman and Kalaba [13, p. 67], $y(t) \geq 0$ for all $t \geq 0$. 
4. $\operatorname{CASE}$ OF $p(t)>0$ AND $q(t)<0$.

If $\mathrm{n}$ is odd, the following two theorems on the number of zeroes of the solution can be obtained.

THEOREM 4.1. If (1) $\mathrm{p}(\mathrm{t})>0$, $\mathrm{a} \leq \mathrm{t} \leq \mathrm{b}$, (2) $\mathrm{q}(\mathrm{t})<0$, $\mathrm{a} \leq \mathrm{t} \leq \mathrm{b}$ and (3) $\mathbf{n}$ is odd, then a necessary condition for $y$ to have two zeroes on $(a, b]$ is that

$$
\int_{a}^{b} p(t) d t>\frac{4}{b-a} .
$$

PROOF. Equation (1.1) can be written in the form

$$
\ddot{y}+\left(p(t)+q(t) y^{n-1}\right) y=0 \text {. }
$$

Let $z(t)$ be real-valued and satisfy the linear equation

$$
\ddot{z}+p(t) z=0 \text {. }
$$

Since $q(t)<0$ and $(n-1)$ is even,

$$
p(t)+q(t) y^{n-1}<p(t)
$$

and by Hartman [12], $z(t)$ has at least two zeroes on $(a, b)$ if $y(t)$ has two zeroes on (a, b]. By Lyapunov Theorem in Hartman [12, p. 346], a necessary condition for $z(t)$ to have two zeroes on $[a, b]$ is that

$$
\int_{a}^{b} p(t) d t>\frac{4}{b-a} .
$$

THEOREM 4.2. If (1) $p(t)>0,0 \leq t \leq T$, (2) $q(t)<0,0 \leq t \leq T$, (3) $n$ is odd, (4) $y(t)$ has $N$ zeroes on $(0, T]$, then

$$
N<\frac{1}{2}\left(T \int_{0}^{T} p(t) d t\right)^{\frac{1}{2}}+1 .
$$


PROOF. As in the proof of Theorem 4.1 , it can be shown that if $z(t)$ has M zeroes on $(0, T)$, then $N \leq M$. But by Hartman [12, p. 346-347],

$$
M<\frac{1}{2}\left(T \int_{0}^{T} p(t) d t\right)^{\frac{1}{2}}+1
$$

and the conclusion follows.

In the next two theorems, $n$ is assumed to be even.

THEOREM 4.3. If (1) $p(t)>0, t \geq 0$, (2) $q(t)<0, t \geq 0$ and (3) $n$ is even, then $y(t) \leq 0$, for $t \geq 0$.

PROOF. Since $q(t)<0$ and $n$ is even,

$$
\ddot{y}+p(t) y+q(t) y^{n} \leq \ddot{y}+p(t) y,
$$

therefore,

$$
0 \leq \ddot{y}+p(t) y
$$

and by Bellman and Kalaba [13, p. 67], $y(t) \leq 0$, for all $t \geq 0$.

If in addition to the hypotheses of Theorem 4.3 , we assume that $\dot{y}(0) \geq 0$, then $y$ is negative and monotonic increasing.

$$
\text { THEOREM 4.4. If (1) } \dot{y}(0) \geq 0, \text { (2) } p(t)>0, t \geq 0, \text { (3) } q(t)<0 \text {, }
$$

$t \geq 0$ and (4) $n$ is even, then $y$ is montonic increasing.

PROOF. Integration of (1.1) from 0 to $t$ leads to

$$
\dot{y}(t)-\dot{y}(0)+\int_{0}^{t} p(s) y d s+\int_{0}^{t} q(s) y^{n} d s=0 .
$$

Since $\mathrm{p}>0$ and $\mathrm{y} \leq 0$ by Theorem 4.3, $\mathrm{q}<0$ and $\mathrm{n}$ is even,

$$
\dot{y}(t)-\dot{y}(0)>\dot{y}(t)-\dot{y}(0)+\int_{0}^{t} p(s) y d s+\int_{0}^{t} q(s) y^{n} d s,
$$


therefore,

$$
\begin{aligned}
& \dot{y}(t)-\dot{y}(0)>0, \\
& \dot{y}(t)>\dot{y}(0) \geq 0
\end{aligned}
$$

and so $\mathrm{y}$ is monotonic increasing.

\section{CASE OF $\mathrm{p}(\mathrm{t})>0$ AND $\mathrm{q}(\mathrm{t})>0$.}

For $p(t)>0, q(t)>0$ and $n$ odd, the following theorems on the oscillatory behavior and boundedness of the solutions can be obtained.

THEOREM 5.1. (1) If $p(t)>0, t \geq 0$, (2) $q(t)>0, t \geq 0$, (3) $n$ is odd and (4) $z(t)$ is a real-valued solution to $\ddot{z}+p(t) z=0$, then $y(t)$ oscillates more rapidly than $z(t)$, for $t \geq 0$.

PROOF. Equation (1.1) can be written in the form

$$
\ddot{y}+\left(p(t)+q(t) y^{n-1}\right) y=0
$$

Let $z(t)$ be a real-valued solution to

$$
\ddot{z}+p(t) z=0
$$

which has been widely discussed. Since $q(t)>0$ and $(n-1)$ is even,

$$
p(t)<p(t)+q(t) y^{n-1}
$$

and the conclusion follows from comparison theorems in Hartman and Sanchez [12, 14].

THEOREM 5.2. If (1) $p(t)>0, \dot{p}(t) \geq 0, t \geq 0$, (2) $q(t)>0, \dot{q}(t) \geq 0$, $t \geq 0$, (3) $n$ is odd and (4) $y$ has successive extrema at $t_{1}, t_{2}, t_{1}<t_{2}$, then $\left|y\left(t_{2}\right)\right| \leq\left|y\left(t_{1}\right)\right|$. (The amplitudes of oscillations do not grow.)

PROOF. The proof is by contradiction, so assume that $\left|y\left(t_{1}\right)\right|<\left|y\left(t_{2}\right)\right|$. Multiplication of $(1.1)$ by $\dot{y}$ leads to

$$
\dddot{y} \dot{y}+p(t) y \dot{y}+q(t) y^{n} \dot{y}=0 .
$$


Integrating (5.1) from $t=t_{1}$ to $t=t_{2}$, we get

$$
\int_{t_{1}}^{t_{2}} p(t) y \dot{y} d t+\int_{t_{1}}^{t_{2}} q(t) y^{n} \dot{y} d t=0
$$

Since

$$
\int_{t_{1}}^{t_{2}} p(t) y \dot{y} d t=\frac{1}{2}\left(p\left(t_{2}\right) y^{2}\left(t_{2}\right)-p\left(t_{1}\right) y^{2}\left(t_{1}\right)-\int_{t_{1}}^{t_{2}} \dot{p}(t) y^{2} d t\right)
$$

and

$$
\int_{t_{1}}^{t_{2}} q(t) y^{n} \dot{y} d t=\frac{1}{n+1}\left(q\left(t_{2}\right) y^{n+1}\left(t_{2}\right)-q\left(t_{1}\right) y^{n+1}\left(t_{1}\right)-\int_{t_{1}}^{t_{2}} \dot{q}(t) y^{n+1} d t\right) \text {, }
$$

(5.2) becomes

$$
\begin{aligned}
& p\left(t_{2}\right) y^{2}\left(t_{2}\right)-p\left(t_{1}\right) y^{2}\left(t_{1}\right)+\frac{2}{n+1} q\left(t_{2}\right) y^{n+1}\left(t_{2}\right)-\frac{2}{n+1} q\left(t_{1}\right) y^{n+1}\left(t_{1}\right) \\
& =\int_{t_{1}}^{t_{2}} \dot{p}(t) y^{2} d t+\frac{2}{n+1} \int_{t_{1}}^{t_{2}} \dot{q}(t) y^{n+1} d t \\
& <y^{2}\left(t_{2}\right)\left(p\left(t_{2}\right)-p\left(t_{1}\right)\right)+\frac{2}{n+1} y^{n+1}\left(t_{2}\right)\left(q\left(t_{2}\right)-q\left(t_{1}\right)\right),
\end{aligned}
$$

therefore

$$
\begin{aligned}
p\left(t_{1}\right)\left(y^{2}\left(t_{2}\right)-y^{2}\left(t_{1}\right)\right) & <\frac{2}{n+1} q\left(t_{1}\right)\left(y^{n+1}\left(t_{1}\right)-y^{n+1}\left(t_{2}\right)\right) \\
& <0 \text {, since } q\left(t_{1}\right)>0 \text { and }(n+1) \text { is even, }
\end{aligned}
$$

which is a contradiction since the left hand side is positive.

THEOREM 5.3. If (1) $\mathrm{p}(\mathrm{t}) \geq 1, \dot{\mathrm{p}}(\mathrm{t}) \leq 0, t \geq 0$, (2) $\mathrm{q}(\mathrm{t})>0, \dot{\mathrm{q}}(\mathrm{t}) \leq 0$ and (3) $n$ is odd, then $y(t)$ is bounded for all $t$. 
PROOF. Multiplication of $(1.1)$ by $\dot{y}$ and integration of the resulting equation from 0 to $t$ lead to

$$
\dot{y}^{2}(t)+p(t) y^{2}(t)-\int_{0}^{t} \dot{p}(s) y^{2} d s+\frac{2}{n+1} q(t) y^{n+1}(t)-\frac{2}{n+1} \int_{0}^{t} \dot{q}(s) y^{n+1} d s=c,
$$

where $C$ is a constant,

therefore

$y^{2}(t)\left(p(t)+\frac{2}{n+1} q(t) y^{n-1}(t)\right)-\int_{0}^{t} \dot{p}(s) y^{2} d s-\frac{2}{n+1} \int_{0}^{t} \dot{q}(s) y^{n+1} d s=c-\dot{y}^{2}(t)$.

Since $\mathrm{p} \geq 1, \mathrm{q}>0, \mathrm{n}$ is odd, $\dot{\mathrm{p}} \leq 0$ and $\dot{\mathrm{q}} \leq 0$, it follows that

$$
y^{2}(t) \leq C, \text { for all } t
$$

and so $\mathrm{y}$ is bounded.

THEOREM 5.4. If (1) $\mathrm{p}(\mathrm{t})>0, \dot{\mathrm{p}}(\mathrm{t}) \geq 0, \mathrm{t} \geq 0$, (2) $\mathrm{q}(\mathrm{t})>0$, $\dot{q}(t) \leq 0, t \geq 0$, and (3) $n$ is odd, then $y(t)$ is bounded for all $t$.

PROOF. As in Theorem 5.3, equation (1.1) is multiplied by $\dot{y}$ and the resulting equation integrated from 0 to $t$, giving,

$\dot{y}^{2}(t)+p(t) y^{2}(t)+\frac{2}{n+1} q(t) y^{n+1}(t)-\frac{2}{n+1} \int_{0}^{t} \dot{q}(s) y^{n+1} d s=c+\int_{0}^{t} \dot{p}(s) y^{2} d s$.

Since $q>0,(n+1)$ is even and $\dot{q} \leq 0$,

so

$$
p(t) y^{2}(t) \leq c+\int_{0}^{t} \dot{p}(s) y^{2} d s
$$

$$
p(t) y^{2}(t) \leq|c|+\int_{0}^{t} p(s) y^{2} \frac{\dot{p}(s)}{p(s)} d s
$$


and by Gronwall's inequality, Hartman [12, p. 24]

$$
\begin{aligned}
p(t) y^{2}(t) & \leq|c| \exp \int_{0}^{t} \frac{\dot{p}(s)}{p(s)} d s \\
& =|c| \frac{p(t)}{p(0)},
\end{aligned}
$$

therefore

$$
y^{2}(t) \leq \frac{|c|}{p(0)} \text { for all } t
$$

In the next theorem, (1.1) is assumed to have constant coefficients $P_{0}$ and $\mathrm{q}_{0} \cdot$

THEOREM 5.5. If (1) $\mathrm{p}_{0}>0$, (2) $\mathrm{q}_{0}>0$ and (3) $\mathrm{n}$ is odd, then $\mathrm{y}$ is oscillatory.

PROOF. The equation is

$$
\ddot{\mathrm{y}}+\mathrm{p}_{0} \mathrm{y}+\mathrm{q}_{0} \mathrm{y}^{\mathrm{n}}=0 \text {. }
$$

By linearization in McLachlan [12, p. 106],

$$
\ddot{\mathrm{y}}+\alpha \mathrm{y}=0 \text {, }
$$

where

$$
\alpha=\frac{1}{\pi A} \int_{0}^{2 \pi}\left(p_{0} A \sin \theta+q_{0} A^{n} \sin ^{n} \theta\right) \sin \theta d \theta,
$$

where $A$ is a constant.

Since $\mathrm{p}_{0}>0, \mathrm{q}_{0}>0$ and $\mathrm{n}$ is odd, the integrand in

$$
\alpha=\frac{1}{\pi} \int_{0}^{2 \pi}\left(p_{0} \sin ^{2} \theta+q_{0} A^{n-1} \sin ^{n+1} \theta\right) d \theta
$$

is positive, so $\alpha>0$. 
By Theorem 5.4, $\mathrm{y}$ is bounded. The fact that

$$
\int_{0}^{\infty} \frac{1}{\alpha} d t=\infty
$$

and $\mathrm{y}$ is bounded imply that $\mathrm{y}$ is oscillatory, see Hartman [12, p. 354].

In the next two theorems, $\mathrm{n}$ is assumed to be even.

THEOREM 5.6. If (1) $p(t)>0, t \geq 0$, (2) $q(t)>0, t \geq 0$ and (3) $n$ is even, then $y(t) \geq 0$, for $t \geq 0$.

PROOF. The equation is

$$
\ddot{y}+p(t) y+q(t) y^{n}=0
$$

Let $y(t)=-z(t)$, then

$$
-\ddot{z}-p(t) z+(-1)^{n} q(t) z^{n}=0 .
$$

Since $\mathrm{n}$ is even,

$$
\ddot{z}+p(t) z-q(t) z^{n}=0
$$

and by Theorem 4.3, $z \leq 0$. Therefore $y(t) \geq 0$ for $t \geq 0$.

If in addition to the hypotheses of Theorem 5.6, we assume that $\dot{y}(0) \leq 0$, then $\mathrm{y}$ is positive and monotonic decreasing.

THEOREM 5.7. If (1) $\dot{y}(0) \leq 0$, (2) $p(t)>0, t \geq 0$, (3) $q(t)>0$, $t \geq 0$ and (4) $\mathrm{n}$ is even, then $\mathrm{y}$ is monotonic decreasing.

PROOF. Integration of (1.1) from 0 to $t$ leads to

$$
\dot{y}(t)-\dot{y}(0)+\int_{0}^{t} p(s) y d s+\int_{0}^{t} q(s) t^{n} d s=0 .
$$

Since $p>0$ and $y \geq 0$ by Theorem 5.6, $q>0$ and $n$ is even, 


$$
\begin{aligned}
& \dot{y}(t)-\dot{y}(0)<0, \\
& \dot{y}(t)<\dot{y}(0) \leq 0
\end{aligned}
$$

and $y$ is monotonic decreasing.

\section{REFERENCES}

1. Ames, W. F. Nonlinear Ordinary Differential Equations in Transport Processes, Academic Press, New York and London, 1968.

2. McLachlan, N. W. Ordinary Non-Linear Differential Equations in Engineering and Physical Sciences, 2nd ed., Oxford University Press, London and New York, 1958.

3. Struble, R. A. Nonlinear Differential Equations, McGraw-Hill Company, New York and London, 1962.

4. Canosa, J. and J. Cole. Asymptotic Behavior of Certain Nonlinear BoundaryValue Problems, J. Math. Phys. 9 (1968) 1915-1921.

5. Canosa, J. Expansion Method for Nonlinear Boundary-Value Problems, J. Math. Phys. 8 (1967) 2180-2183.

6. Skidmore, A. On the Differential Equation $y^{\prime \prime}+p(x) y=f(x)$, J. Math. Ana1. App1. 43 (1973) 46-55.

7. Abramovich, S. On the Behavior of the Solutions of $y^{\prime \prime}+p(x) y=f(x)$, J. Math. Anal. App1. 52 (1975) 465-470.

8. Rankin, S. M. Oscillation Theorems for Second-Order Nonhomogeneous Linear Differential Equations, J. Math. Anal. App1. 53 (1976) 550-553.

9. Grimmer, R. C. and W. T. Patula. Nonoscillatory Solutions of Forced SecondOrder Linear Equations, J. Math. Anal. App1. 56 (1976) 452-459.

10. Chen, L. S. Some Oscillation Theorem for Differential Equations with Functional Arguments, J. Math. Anal. App1. 58 (1977) 83-87.

11. Chen, M. P., C. C. Yeh and C. S. Yu. Asymptotic Behavior of Nonoscillatory Solutions of Nonlinear Differential Equations with Retarded Arguments, J. Math. Ana1. App1. 59 (1977) 211-215.

12. Hartman, P. Ordinary Differential Equations, John Wiley and Sons, New York and London, 1964. 
13. Bellman, R. E. and R. E. Kalaba. Quasilinearization and Nonlinear BoundaryValue Problems, American Elsevier Publishing Company, New York, 1965.

14. Sanchez, D. A. Ordinary Differential Equations and Stability Theory,

W. H. Freeman and Company, San Francisco and London, 1968.

KEY WORDS AND PHRASES. Nonlinear differential equations, oscillatory and asymptotic behavior of solutions, boundedness and monotonicity of solutions.

AMS (MOS) SUBJECT CLASSIFICATIONS (1970). 34C10, 34C15, 34005. 


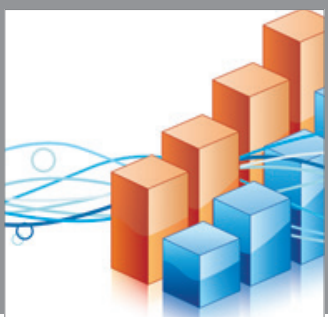

Advances in

Operations Research

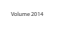

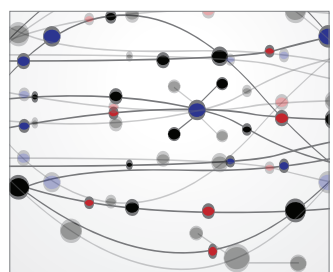

\section{The Scientific} World Journal
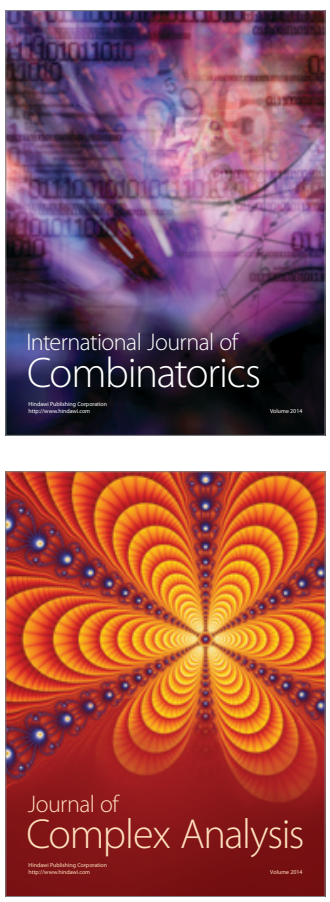

International Journal of

Mathematics and

Mathematical

Sciences
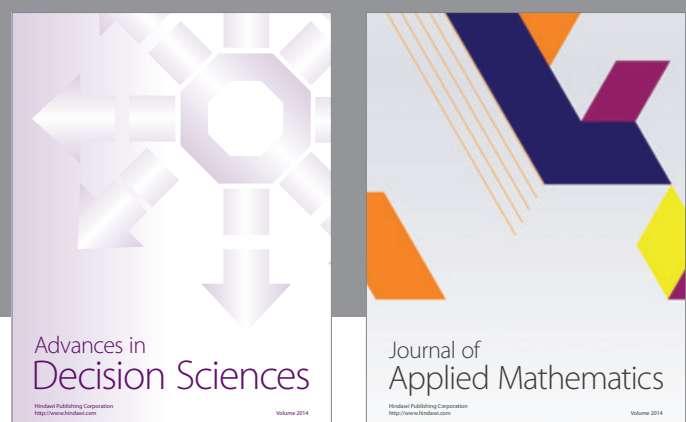

Journal of

Applied Mathematics
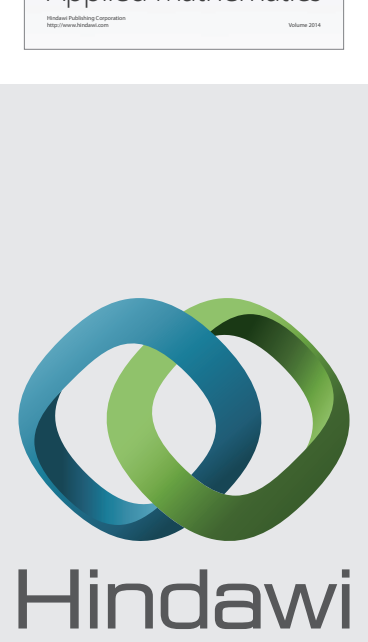

Submit your manuscripts at http://www.hindawi.com
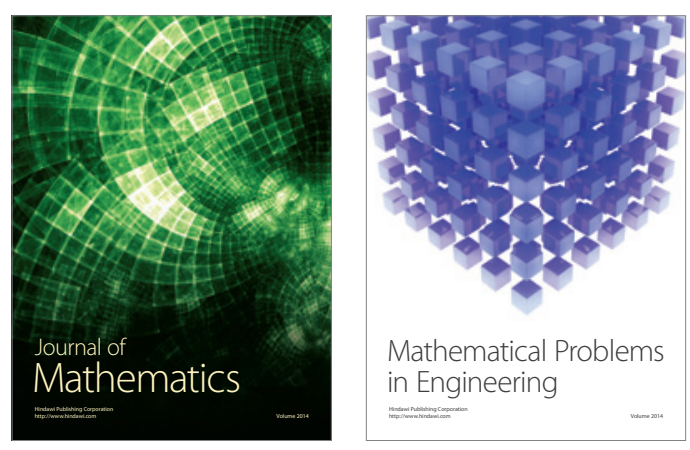

Mathematical Problems in Engineering
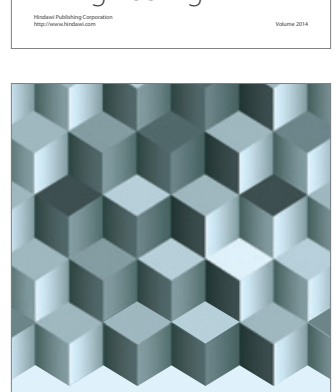

Journal of

Function Spaces
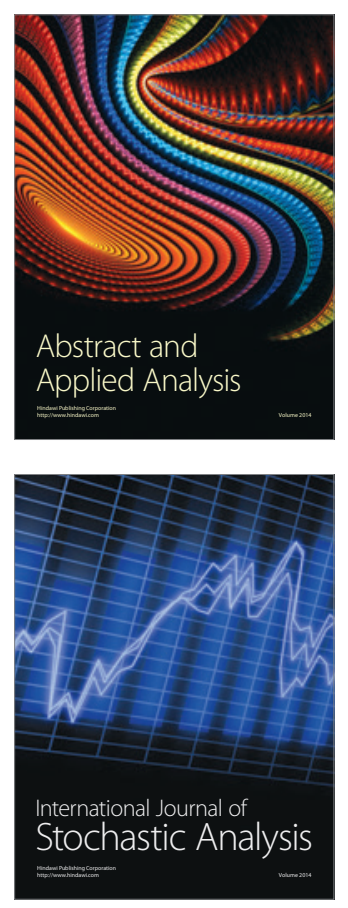

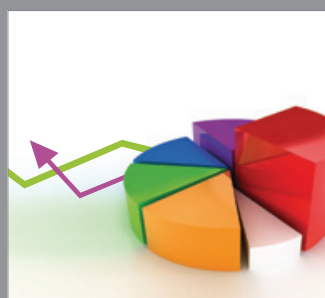

ournal of

Probability and Statistics

Promensencen
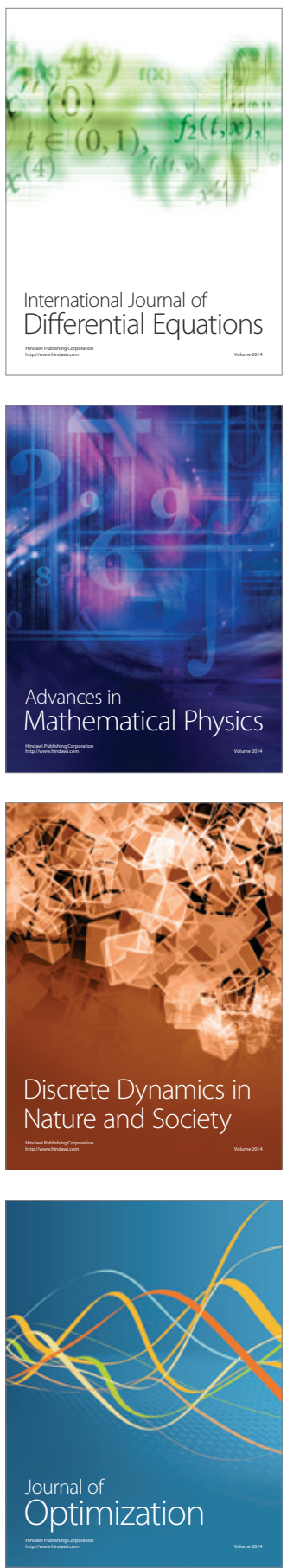